\title{
TB Elimination Requires Discovery and Development of Transformational Agents
}

\author{
Christian Lienhardt ${ }^{1}$ and Mario C. Raviglione ${ }^{2, *}$ \\ 1 Unité Mixte Internationale TransVIHMI (UMI 233 IRD-U1175 INSERM, Université de Montpellier)-Institut \\ de Recherche pour le Développement (IRD), 34000 Montpellier, France; christian.lienhardt@ird.fr \\ 2 Centre for Multidisciplinary Research in Health Science (MACH), Università di Milano, Milan, Italy and \\ Global Studies Institute, Université de Genève, 1211 Genève, Switzerland \\ * Correspondence: mario.raviglione@unimi.it
}

Received: 24 March 2020; Accepted: 7 April 2020; Published: 10 April 2020

check for updates

\begin{abstract}
The World Health Organization (WHO) End Tuberculosis (TB) Strategy has set ambitious targets to reduce 2015 TB incidence and deaths by $80 \%$ and $90 \%$, respectively, by the year 2030 . Given the current rate of TB incidence decline (about $2 \%$ per year annually), reaching these targets will require new transformational tools and innovative ways to deliver them. In addition to improved tests for early and rapid detection of TB and universal drug-susceptibility testing, as well as novel vaccines for improved prevention, better, safer, shorter and more efficacious treatments for all forms of TB are needed. Only a handful of new drugs are currently in phase II or III clinical trials, and a few combination regimens are being tested, mainly for drug-resistant TB. In this article, capitalising on an increasingly rich medicine pipeline and taking advantage of new methodological designs with great potential, the main areas where progress is needed for a transformational improvement of treatment of all forms of TB are described.
\end{abstract}

Keywords: tuberculosis treatment; biomarkers; drug combination; clinical trial

On 14 May 2014, the 67th World Health Assembly (WHA) endorsed a resolution detailing the global strategy to control and eliminate tuberculosis (TB) in the 2015-2030 Sustainable Development Goal (SDG) era [1]. The 3-pillar strategy was branded as the "End TB Strategy" [2]. Additional to two pillars devoted to patient-centred care and to health system policies, a third pillar is fully devoted to research and the need of innovations. This pillar and its components are fundamental in reaching ambitious international targets set as part of the new strategy to "End TB": to reduce 2015 TB incidence and deaths by $80 \%$ and $90 \%$, respectively, by the year 2030 . The simple projection model underpinning such figures, presented at the 67th WHA, is based on previous empirical experiences showing the plausibility of declining trends conducive to those targets. The large-scale interventions promoted by the End TB Strategy would be able to reduce TB incidence at a much higher annual rate than the current $1.5-2 \%$ per year [3]. For instance, it is known that in the Netherlands, United Kingdom, Germany and other western European countries, TB incidence was declining at $8 \%-10 \%$ per year during the late 1950s and 1960s thanks to wide access to diagnosis and effective chemotherapy, screening of people at risk, affordable care and social protection mechanisms [4]. It is also known that additional intensive interventions, including large-scale preventive therapy, were associated with even faster declines reaching $17 \%$ per year among the small Inuit populations of Alaska and North-Western Territory of Canada [5]. However, while these declines could in theory be achieved with optimal implementation of existing diagnostic and treatment tools — which are better than those available 70 years ago- the needed acceleration towards $15 \%-20 \%$ incidence decline per year to reach the 2030 targets will require new transformational tools and innovative ways to deliver them. These tools need to cover all aspects of TB care and its cascade, as well as prevention. 


\section{Which New Tools Are Necessary to End the Tuberculosis (TB) Epidemic?}

First of all, achieving the End TB targets will require improved tests for early and rapid detection of TB and for universal drug-susceptibility testing (DST) to reach more patients when they first seek care so that one can cut transmission early and accelerate the decline in TB incidence and mortality. Diagnostics must be rapid, precise, connectable, available at the point-of-care and effective in detecting both active disease and latent infection [6]. Molecular diagnostics and sequencing technology available today are powerful tools, but still need relatively sophisticated laboratory capacity, and are not offering a point of care solution thus limiting their efficacy in the field.

Second, we need better, safer, shorter and more efficacious treatment for all forms of TB. The current research and development pipeline-although the most populated seen in the past few decades-still shows only less than 10 new agents in phase 1 trials, a handful of new drugs in phase 2 or 3 trials, and a few combination regimens being tested mainly for drug-resistant TB [7]. Completely new classes of anti-TB compounds are scarce, and potential synergistic combinations still unknown. Furthermore, targeting better treatment of active TB towards elimination goals is clearly not enough, as the reservoir of latent TB infection needs to be tackled as well. Mathematical models show that elimination may not be possible without targeting simultaneously latent infection and active disease [8]. Thus, addressing the vast pool of at-risk individuals among the estimated 1.7 billion people who may be latently infected is paramount, and solutions must be found that are simple and can be implemented safely among those at the highest risk of disease. Currently, the pipeline for treatment of latent infection includes a few trials using essentially known drugs such as isoniazid, rifampicin and rifapentine in different combinations, dosage and duration.

Lastly, a vaccine would be the ultimate solution if found to be highly effective, safe, able to prevent pre-exposure infection as well as reactivation. However, at the moment, the most advanced and promising vaccine candidate among the 14 in the current pipeline offers at best a $50 \%$ protection among those with latent infection, and this still needs confirmation [9]. A fully effective potent vaccine is not envisaged for several years, and will certainly not be available on time to allow reaching the 2030 targets.

Notwithstanding the importance of new, rapid diagnostics and efficacious vaccines, efforts to populate the pipeline and accelerate anti-TB drug research are a top priority for investors and researchers alike. Lately, after several years of stagnation, financial investments have been growing slowly passing 800 million US\$ in 2019 [10]. However, this is far from sufficient to truly accelerate research toward new agents and regimens. The modest target of 2 billion US\$/year, promoted within an existing international plan [11], is not at reach at the moment.

At the same time, efforts in drug research begun in the early 2000s resulted in some successes with the discovery and development of agents such as bedaquiline and delamanid that are recommended for the treatment of drug-resistant TB. Recently, the combination of two new drugs-pretomanid and bedaquiline-with a re-purposed agent, linezolid, has resulted in unprecedented high rates of cure among advanced forms of multidrug-resistant TB (MDR-TB) and of extensively drug-resistant TB (XDR-TB) (Nix-TB trial) [12]. The demonstrated 90\% cure rates observed in a single-arm, open-label trial in South Africa has prompted approval by the US Food and Drug Administration (FDA), despite the high frequency of adverse events, including bone marrow suppression and peripheral neuropathy, due to linezolid. These advances show that with wise investments and a well-thought strategy that pursues development of a full regimen composed of new and existing or re-purposed drugs rather than of individual new drugs-as promoted by the WHO Target Regimen Profiles [13] -may be conducive to success despite the obvious challenges that TB research poses. This now needs to be consolidated and accelerated, so that we can hope to obtain a shorter and safer novel regimen that can treat TB irrespective of pre-existing drug resistance (and thus with reduced need for drug-resistance testing). 


\section{Which New Drugs and Regimens?}

First, further progress would require improving molecules of known classes. The Nix-TB trial has raised much hope for the development of a short fully oral regimen for the treatment of severe drug-resistant TB. The use of the tested regimen may, however, be limited in clinical practice due to severe side-effects caused by linezolid. While a trial is on-going to test lower dose of linezolid, [14] new oxazolidinones are being developed to try and reduce toxic effects. Four oxazolidinones are now in early phases of the clinical development pipeline, i.e., contezolid, delpazolid, sutezolid and TBI-223 [7]. Similarly, the use of clofazimine, another important component of the therapeutic armamentarium against drug-resistant TB, is hampered by potential undesirable skin pigmentation. Novel riminophenazine derivatives are being developed with the goal of maintaining potent anti-tuberculosis activity while lowering side effects-such as TBI-166, a compound currently in clinical development in China [15].

The second requirement is that of developing new agents or, better classes of anti-TB drugs.

While improving existing classes has the advantage of initiating the risky discovery process with a well-characterized, validated compound, some level fast adaptation of bacterial populations can be expected. Ideally, therefore, research and development should produce entirely new classes, targets and modes of action to avoid cross-resistance to existing antibiotics. Thus, in parallel with improvements in existing drug and regimen models, innovative approaches, including discovery of novel chemical scaffolds and identification of new targets, are urgently needed.

The discovery of bedaquiline opened the way to investigating the possibility to alter the energy metabolism in mycobacteria, in particular the oxidative phosphorylation pathway, as a novel target pathway in drug discovery, leading to the depletion of ATP synthesis of M. tuberculosis. New classes of antibacterial drugs interfering with elements of this pathway have been shown to be highly active in combating latent mycobacterial infections. The discovery of Q203, a candidate drug targeting the cytochrome bc1 complex in the respiratory chain, has highlighted the importance of this new target pathway. Inhibiting the bacterial energy metabolism might be a key feature of novel and sterilizing drug combinations for the treatment of TB [16]. Furthermore, Q203 was shown to have good synergy with bedaquiline in the murine chronic infection model, indicating promising potential for new treatment regimens [17]. Thus, the combination of drugs targeting various elements of the oxidative phosphorylation pathway could lead to a completely new regimen for drug-susceptible and multi-drug resistant tuberculosis.

A series of new compounds are currently developed that focus on newly identified targets. Four of these inhibit DprE1 (decaprenylphosphoryl- $\beta$-D-ribose 2-epimerase), a flavoenzyme that catalyses a key step in the synthesis of the complex cell wall of M. tuberculosis: macozinone, BTZ-043, OPC-167832 and TBA-7371 [7]. Another compound targets the leucyl-tRNA synthetase (LeuRS), which is essential for protein synthesis (GSK3036656).

The third approach is to identify efficient and seamless development processes to accelerate testing of novel treatment regimens. The current TB treatment development pathway is complex, lengthy and costly, partly due to the fact that some drugs are still being developed individually, and partly due to the lack of reliable surrogate markers of treatment outcomes and the lack of predictive quantitative relationships between Phase II and Phase III outcomes [18]. Currently, Phase II TB drug development includes 14-day early bactericidal activity monotherapy studies to identify the maximally efficacious dose for a new chemical entity (Phase IIA), followed by 2-month serial sputum colony count studies (Phase IIB), in which the efficacy of treatment combinations is usually studied with time-to-sputum-culture-conversion as the primary endpoint. In addition to long duration, this approach suffers multiple weaknesses, including an inadequate exploration of dose-response, the lack of means to determine early the optimal combination of drugs to be tested and the optimal duration of therapy, as well as the inability to study multiple regimens in parallel [19].

Research is needed to identify biomarkers that could predict relapse and guide selection of suitable drug combination(s) and treatment duration(s) so as to accelerate drug development in TB. Novel 
approaches are being explored to identify early and streamline suitable drug combinations to advance from early to late phases of development taking into account new developments in pharmacokinetic and pharmacodynamic methodology and modelling [20], as well as novel adaptive designs [21]. The "multi-arm multi-stage" (MAMS) design, initially used in oncology, allows testing a broad range of combinations and dose levels without requiring a large sample size, dropping early arms that do not meet pre-specified efficacy threshold [22]. Recently, a newer approach has been proposed that combines Phase II and Phase III features. In this design, named "selection trial with extended post-treatment follow-up" (STEP), limited long-term follow-up data on relapse are collected, together with data on culture conversion, permitting estimation of a Bayesian prediction interval for the likely results of a future Phase III trial [23]. Such Phase IIB/C studies, with arms testing different doses and durations, coupled with the use of novel biomarkers for sterilising cures-these being either RNA expression, cytokine, bacterial or radiological markers-would strengthen and accelerate the process for identifying candidate regimens likely to succeed in Phase III [24], as well as prospectively validating novel biomarkers against the relapse endpoint.

\section{Conclusions}

We are now at a crossroads in new anti-tuberculosis drug and regimen development. New initiatives, such as the the European Union (EU) Innovative Medicines Initiative IMI2 call and the Gates Medical Research Institute alliance, have the potential to accelerate research and synergize with existing efforts carried out by academia, public-private partnerships, such as the TB Alliance, and industry. If researchers collaborate and join forces towards the common aim of innovative regimens, there is a possibility of pursuing effectively a transformational improvement of treatment of all forms of TB [25]. If, however, such initiatives compete for limited resources and resist cooperation, the targets expressed at the time of the Moscow Conference in late 2017 and subsequently at the United Nations General Assembly in 2018 may remain a dream.

Author Contributions: The two authors contributed equally to the writing of the article. All authors have read and agreed to the published version of the manuscript.

Funding: This research received no external funding.

Conflicts of Interest: The authors declare no conflict of interest.

\section{References}

1. World Health Organization. Sixty-Seventh World Health Assembly WHA67.1; World Health Organization: Geneva, Switzerland, 2014.

2. World Health Organization. Global Strategy and Targets for Tuberculosis Prevention, Care and Control after 2015; Agenda item 12.1, 21 May 2014; World Health Organization: Geneva, Switzerland, 2014.

3. Uplekar, M.; Weil, D.; Lönnroth, K.; Jaramillo, E.; Lienhardt, C.; Dias, H.M.; Falzon, D.; Floyd, K.; Gargioni, G.; Getahun, H.; et al. WHO's End TB Strategy. Lancet 2015, 385, 1799-1801. [CrossRef]

4. World Health Organization. Global Tuberculosis Report 2019; World Health Organization: Geneva, Switzerland, 2019.

5. Styblo, K. Epidemiology of Tuberculosis; The Royal Netherlands Tuberculosis Association (KNCV): The Hague, The Netherlands, 1991.

6. Grzybowski, S.; Styblo, K.; Dorken, E. Tuberculosis in Eskimos. Tubercle 1976, 57, 1-58. [CrossRef]

7. Walzl, G.; McNerney, R.; du Plessis, N.; Bates, M.; McHugh, T.D.; Chegou, N.N.; Zumla, A. Tuberculosis: Advances and challenges in development of new diagnostics and biomarkers. Lancet Infect Dis. 2018, 18, e199-e210. [CrossRef]

8. Stop TB Partnership's Working Group on New TB Drugs Clinical Pipeline. Available online: https: //www.newtbdrugs.org/pipeline/clinical (accessed on 30 March 2020).

9. Dye, C.; Williams, B.G. Eliminating human tuberculosis in the 21st century. J. R. Soc. Interface 2008, 5, 653-662. [CrossRef] [PubMed] 
10. Tait, D.R.; Hatherill, M.; Van Der Meeren, O.; Ginsberg, A.M.; Van Brakel, E.; Salaun, B.; Scriba, T.J.; Akite, E.J.; Ayles, H.M.; Bollaerts, A.; et al. Final Analysis of a Trial of M72/AS01E Vaccine to Prevent Tuberculosis. N. Engl. J. Med. 2019, 381, 2429-2439. [CrossRef]

11. Treatment Action Group. Tuberculosis Research Funding Trends 2005-2018; Treatment Action Group: New York, NY, USA, 2019; ISBN 978-0-9983966-8-2.

12. The Lancet Commission on TB. Building a Tuberculosis-Free World: The Lancet Commission on Tuberculosis. Lancet 2019. [CrossRef]

13. Conradie, F.; Diacon, A.H.; Ngubane, N.; Howell, P.; Everitt, D.; Crook, A.M.; Mendel, C.M.; Egizi, E.; Moreira, J.; Timm, J.; et al. Treatment of Highly Drug-Resistant Pulmonary Tuberculosis. N. Engl. J. Med. 2020, 382, 893-902. [CrossRef]

14. World Health Organization. Target Regimen Profiles for TB Treatment: Candidates: rifampicin-Susceptible, Rifampicinresistant and pan-TB Treatment Regimens; World Health Organization: Geneva, Switzerland, 2016.

15. Zhang, D.; Liu, Y.; Zhang, C.; Zhang, H.; Wang, B.; Xu, J.; Fu, L.; Yin, D.; Cooper, C.B.; Ma, Z.; et al. Synthesis and biological evaluation of novel 2-methoxypyridylamino-substituted riminophenazine derivatives as antituberculosis agents. Molecules 2014, 19, 4380-4394. [CrossRef]

16. Bald, D.; Villellas, C.; Lu, P.; Koul, A. Targeting energy metabolism in Mycobacterium tuberculosis, a new paradigm in antimycobacterial drug discovery. mBio 2017, 8, e00272-17. [CrossRef]

17. Shi-Yan Foo, C.; Pethe, K.; Lupien, A. Oxidative Phosphorylation-an Update on a New, Essential Target Space for Drug Discovery in Mycobacterium tuberculosis. Appl. Sci. 2020, 10, 2339. [CrossRef]

18. Ginsberg, A.M.; Spigelman, M. Challenges in tuberculosis drug research and development. Nat Med. 2007, 13, 290-294. [CrossRef]

19. Lienhardt, C.; Nahid, P. Advances in clinical trial design for development of new TB treatments: A call for innovation. PLoS Med. 2019, 16, e1002769. [CrossRef]

20. Dooley, K.E.; Hanna, D.; Mave, V.; Eisenach, K.; Savic, R.M. Advancing the development of new tuberculosis treatment regimens: The essential role of translational and clinical pharmacology and microbiology. PLoS Med. 2019, 16, e1002842. [CrossRef] [PubMed]

21. Davies, G.R.; Phillips, P.P.J.; Jaki, T. Adaptive clinical trials in tuberculosis: Applications, challenges and solutions. Int. J. Tuberc. Lung Dis. 2015, 19, 626-634. [CrossRef]

22. Boeree, M.J.; Heinrich, N.; Aarnoutse, R.; Diacon, A.H.; Dawson, R.; Rehal, S.; Kibiki, G.S.; Churchyard, G.; Sanne, I.; Ntinginya, N.E.; et al. High-dose rifampicin, moxifloxacin, and SQ109 for treating tuberculosis: A multi-arm, multi-stage randomised controlled trial. Lancet Infect Dis. 2017, 17, 39-49. [CrossRef]

23. Phillips, P.P.J.; Dooley, K.E.; Gillespie, S.H.; Heinrich, N.; Stout, J.E.; Nahid, P.; Diacon, A.H.; Aarnoutse, R.E.; Kibiki, G.S.; Boeree, M.J.; et al. A new trial design to accelerate tuberculosis drug development: The Phase IIC Selection Trial with Extended Post treatment follow-up (STEP). BMC Med. 2016, 14, 51. [CrossRef] [PubMed]

24. Davies, G.; Hoelscher, M.; Boeree, M.; Hermann, D. Accelerating the transition of new tuberculosis drug combinations from Phase II to Phase III trials: New technologies and innovative designs. PLoS Med. 2019, 16, e1002851. [CrossRef]

25. Lienhardt, C.; Nunn, A.; Chaisson, R.; Vernon, A.A.; Zignol, M.; Nahid, P.; Delaporte, E.; Kasaeva, T. Advances in clinical trial design: Weaving tomorrow's TB treatments. PLoS Med. 2020, 17, e1003059. [CrossRef]

(C) 2020 by the authors. Licensee MDPI, Basel, Switzerland. This article is an open access article distributed under the terms and conditions of the Creative Commons Attribution (CC BY) license (http://creativecommons.org/licenses/by/4.0/). 